\title{
TERAPIA DIALÉCTICO CONDUCTUAL PARA EL TRASTORNO DE PERSONALIDAD LÍMITE \\ DIALECTICAL BEHAVIORAL THERAPY IN BORDERLINE PERSONALITY DISORDER
}

\author{
IRENe de La Vega ${ }^{a}$ Y SABrina SÁnchez Quintero ${ }^{b}$ \\ a Instituto de Psiquiatría y Salud Mental. Hospital Clínico San Carlos. Madrid. irenevr@hotmail.com \\ b Centro de Atención Integral a Drogodependientes (CAID Sur). Agencia Antidroga. Madrid
}

Cómo referenciar este artículo/How to reference this article:

De la Vega-Rodríguez, I. y Sánchez-Quintero, S. (2013). Terapia dialéctico conductual para el trastorno de personalidad límite [Dialectical behavioral therapy in borderline personality disorder]. Acción Psicológica, 10(1), 45-56. http://dx.doi.org/10.5944/ap.10.1.7032

\section{Resumen}

La terapia dialéctico conductual (TDC) es una terapia psicológica de tercera generación desarrollada por Marsha Linehan (1993a, 1993b) para tratar específicamente los síntomas característicos del trastorno límite de la personalidad: inestabilidad afectiva, trastorno de identidad, impulsividad y dificultades en las relaciones sociales. La TDC parte de un modelo biosocial del trastorno límite y combina técnicas cognitivo conductuales con aproximaciones zen y budistas basadas en la aceptación de la realidad tal como se presenta. El tratamiento consiste en un entrenamiento en habilidades, psicoterapia individual y atención en crisis y es llevado a cabo por un equipo coordinado de profesionales que ejercen distintas funciones. La TDC cuenta con estudios de eficacia y se está aplicando con éxito a otras poblaciones de pacientes con elevada impulsividad.

Palabras clave: Trastorno límite de la personalidad; Terapia dialéctico conductual; Terapia cognitivo conductual; Disregulación emocional.

\begin{abstract}
Dialectical behavior therapy (DBT) is a third generation psychological therapy developed by Marsha Linehan (1993a, 1993b) to specifically manage the characteristic symptoms of borderline personality disorder: affective instability, identity disorder, impulsivity and difficulties in social relationships. The DBT comes from a biosocial model of BPD and combine cognitive behavioral techniques with Zen and Buddhist concepts based on an acceptance of reality as presented. The treatment consists of skills training, individual psychotherapy and crisis intervention and it is carried out by a coordinated team of professionals engaged in different functions. The DBT has efficacy studies and it is being successfully applied to other populations of patients with high impulsivity.
\end{abstract}

Keywords: Borderline personality disorder; Dialectical behavior therapy; Cognitive behavioral therapy; Emotional dysregulation. 


\section{Introducción}

La terapia dialéctico conductual (TDC) (Linehan, 1993a, 1993b) es un tratamiento psicológico desarrollado específicamente para abordar el Trastorno Límite de Personalidad, especialmente los síntomas de impulsividad e inestabilidad que se materializan en actos suicidas y parasuicidas. La TDC pertenece a la llamada tercera generación de terapias psicológicas que, haciendo énfasis en el contexto y la función, incorporan a los planteamientos conductuales y cognitivos clásicos conceptos como la atención plena, los valores, o la aceptación. (Hayes, Villate, Levin y Hildebrandt, 2011).

La TDC tiene una filosofía integradora, combinando elementos cognitivo conductuales con aproximaciones de aceptación del sufrimiento basadas en filosofías zen y budistas. El tratamiento original de la TDC, tal como fue desarrollado por la Dra. Marsha Linehan (1993a, 1993b) y su grupo de investigación en la Universidad de Washington (EEUU) consiste en un paquete que incluye psicoterapia individual, entrenamiento en habilidades, atención telefónica de las crisis y reuniones periódicas de supervisión.

Desde la perspectiva de la TDC la disfunción principal de los pacientes límite es la incapacidad para regular el afecto, incapacidad de la que se derivan la mayor parte de los síntomas característicos del TLP. En concreto, la TDC actúa sobre cuatro grupos sintomáticos habitualmente presentes en estos pacientes: confusión acerca del yo, impulsividad, caos interpersonal e inestabilidad emocional. El objetivo ayudar al paciente a poner en marcha conductas funcionales y adaptadas, incluso cuando está experimentando emociones muy intensas. (Lynch, Chapman, Rosenthal, Kuo y Linehan, 2006). El tratamiento, que tendría al menos dos años de duración, culminaría cuando el paciente fuera capaz de construir «una vida que merezca la pena vivir» Las técnicas empleadas incluyen procedimientos de exposición, moldeamiento, aprendizaje por modelos, rol playing, instrucciones y autoinstrucciones, refuerzos y feedback y se trabaja fundamentalmente validando la experiencia emocional del paciente, con una perspectiva no enjuiciadora.

\section{Aspectos básicos de la TDC}

\section{Visión dialéctica del mundo}

La perspectiva dialéctica es uno de los aspectos más característicos de la TDC, y supone un factor diferenciador respecto a otras terapias cognitivo conductuales (Chapman, 2006). La visión dialéctica de la realidad, el mundo y la conducta puede sintetizarse en los siguientes puntos (Linehan, 1993b):

1. La realidad está formada de partes que se interrelacionadas y que funcionan como un todo: las conductas de un individuo no pueden entenderse de forma individual, sino como partes de un amplio repertorio dentro de un ambiente complejo. Si un sujeto aprende habilidades, por tanto, deberá aprender también otras relacionadas y capacitarse, al mismo tiempo, para influir sobre el ambiente para poder llevar a cabo su aprendizaje.

2. La realidad es un conjunto de fuerzas que se oponen entre sí. El sujeto con TLP está atrapado en polaridades y es incapaz de conseguir la síntesis. El terapeuta debe ayudar al paciente a lograr una síntesis viable, entre, por ejemplo, su necesidad de aceptarse a sí mismo tal cual es y su necesidad de cambiar.

3. La naturaleza fundamental de la realidad es el cambio o proceso, el individuo y el ambiente llevan a cabo una transacción continua; la terapia se concentra en ayudar al paciente a estar cómodo con el cambio.

\section{Modelo biosocial del trastorno límite}

El modelo etiológico propuesto por Linehan (1993a), de tipo biosocial, es una de las teorías mejor articuladas sobre el origen del 
TPL. Según este modelo, la disregulación emocional, disfunción principal de los sujetos con TLP, es el resultado de la interacción entre una predisposición biológica a la vulnerabilidad emocional y un ambiente invalidante. Así, el niño que ha nacido biológicamente vulnerable crece en un ambiente que responde de forma errática o exagerada a sus eventos internos (emociones, sensaciones, preferencias...), negándolos («deja de llorar»; "¿cómo vas a tener sed, si acabas de beber?») o atribuyéndolos a rasgos negativos de la personalidad («eres un llorón»; «no seas cobarde»). Las familias invalidantes restringen las demandas del niño, no tienen en cuenta sus preferencias («qué sabrás tú») y utilizan el castigo como forma de controlar la conducta. En este ambiente, el niño no aprende a poner nombre a sus emociones, modularlas, tolerar el malestar o confiar en sus repuestas emocionales como interpretaciones válidas de los eventos. Como consecuencia, durante su infancia y la vida adulta, estos sujetos tienen mayor sensibilidad emocional, dificultades para modular el afecto y un lento retorno a la línea base, lo que dificulta seriamente su capacidad de adaptación vital y da lugar a la mayor parte de síntomas y signos característicos del TLP. Para profundizar sobre el desarrollo reciente del modelo biosocial del trastorno límite remitimos al lector al magnífico trabajo de Crowell, Beauchaine y Linehan (2009).

\section{Estructura de la TDC}

Toda la estructura y aplicación de la TDC puede consultarse en los manuales de referencia de la autora (Linehan, 1993a y Linehan 1993b). Exponemos a continuación un resumen de los aspectos más relevantes recogidos en dichos textos.

La TDC es una terapia ampliamente organizada, que incluye trabajo entre sesiones y tareas para casa. La directividad por parte de los terapeutas es mayor en las etapas iniciales, cuando los pacientes carecen de control conductual y se implican en conductas que ponen en riesgo su propia vida.
La meta fundamental del tratamiento es que el paciente incorpore en su repertorio habilidades que permitan regular sus emociones y su conducta. Esto requiere que tanto el paciente como el terapeuta se ciñan a una disciplina que facilite la consecución de objetivos. Sin embargo, ceñirse a una disciplina puede resultar complicado cuando se trata de pacientes con personalidad límite: las frecuentes crisis y la intolerancia al malestar hacen que el paciente difícilmente se pueda concentrar en el aprendizaje y el terapeuta muchas veces se ve obligado a posponer el plan de la sesión para atender al malestar y sufrimiento actual. Debido a esto, el tratamiento se divide en dos componentes principales: un grupo de entrenamiento específico en habilidades, por un lado, y por otro una terapia individual, donde hacer que el individuo saque a la luz las habilidades aprendidas y donde se puede atender y dar respuesta a las crisis del momento, así como a otras conductas que interfieren en el curso del tratamiento.

Es importante que estas dos partes del tratamiento estén claramente diferenciadas. El entrenamiento en habilidades se hará de forma grupal y puede ser llevado a cabo por un terapeuta distinto al que conduce la psicoterapia individual. Si esto no es posible, el terapeuta individual que quiera formar a su paciente en habilidades deberá conseguir que el contexto del programa de formación sea diferente del contexto de la psicoterapia normal, planificando un encuentro separado sólo destinado a las habilidades, cambiando de sala o cambiando la disposición del despacho, la iluminación o los elementos (por ejemplo, añadiendo una pizarra). El distinto encuadre facilitará que tanto el paciente como el terapeuta puedan concentrarse en el entrenamiento de habilidades, y dejar otros temas para el momento de la psicoterapia individual.

\section{EI entrenamiento en habilidades}

El entrenamiento en habilidades es un aspecto central de la TDC, que, idealmente, es llevado a cabo por dos terapeutas en una sesión grupal de unas dos horas y media de duración. 
El encuadre grupal facilita que el terapeuta se muestre lo bastante directivo como para llevar a cabo la sesión y que no se deje llevar por los problemas urgentes de los pacientes. Además, la terapia no se ve interferida cuando un paciente en concreto no está de humor para la formación, ya que se cuenta con el interés del resto del grupo. Por otro lado, el grupo permite observar y trabajar las conductas interpersonales que surgen entre los participantes, proporciona apoyo y permite que los pacientes aprendan unos de otros, aumentando las fuentes de aprendizaje. Los pacientes suelen encontrar este formato educativo más útil y menos estresante que otros grupos destinados a la descarga emocional.

Contar con un segundo terapeuta de apoyo en el grupo de entrenamiento minimiza el des- gaste asociado a este tipo de grupo. Es muy importante que los responsables del grupo se reúnan con frecuencia con el terapeuta individual, para garantizar el avance del tratamiento.

La TDC se compone de cuatro módulos de habilidades específicas, basadas en las dificultades de regulación de los pacientes y sus consecuencias: habilidades básicas de conciencia, habilidades de efectividad interpersonal, habilidades de regulación de las emociones y habilidades de tolerancia al malestar. Cada módulo requiere alrededor de ocho sesiones de entrenamiento (tabla 1). El primer módulo, habilidades básicas de conciencia, puede requerir unas tres sesiones pero se repasa y refuerza al principio antes de cada nuevo módulo.

Tabla 1

Estructura del entrenamiento en habilidades en la TDC

\begin{tabular}{ll}
\hline \multicolumn{1}{c}{ Módulo de entrenamiento } & \multicolumn{1}{c}{ Síntomas diana } \\
\hline Habilidades básicas de conciencia & Confusión acerca del yo. Identidad. Vacío \\
Habilidades de efectividad interpersonal & Caos interpersonal. Relaciones inestables. \\
Habilidades de regulación emocional & Inestabilidad emocional y de estados de ánimo \\
Habilidades de tolerancia al malestar & Impulsividad \\
\hline
\end{tabular}

\section{A. Habilidades básicas de conciencia}

La incorporación de técnicas de mindfulness a la psicoterapia ha supuesto toda una revolución y está consiguiendo resultados muy prometedores en el tratamiento de diversas patologías. (Schnyder, 2009). En la TDC, las técnicas de mindfulness se explican y entrenan de forma específica en el módulo de habilidades básicas de conciencia, aunque están presentes a lo largo de toda la formación.

Las habilidades básicas de conciencia son las primeras que se enseñan y su entrenamiento se trabaja durante todo el tratamiento por medio de fichas que los pacientes rellenan cada semana. Estas habilidades están basadas en las filosofías orientales de meditación, y van especialmente dirigidas a los sentimientos de vacío y desconocimiento del yo.

El objetivo, tal como se expone a los pacientes, es: «Aprender a controlar nuestra propia mente, en vez de dejar que la mente nos controle a nosotros.» Controlar la mente implica, en cierta medida, controlar los procesos atenciones, decidir qué se atiende y durante cuánto tiempo se atiende. Los ejercicios de mindfulness ayudan a que el paciente concentre su atención en el «aquí y ahora».

En este módulo se plantean tres estados mentales fundamentales: 1 . la mente racional; que piensa de forma lógica y fría, 2. la mente emocional, que actúa en caliente, dejándose 
guiar por las emociones del momento, y 3. la mente sabia, que integra las dos anteriores. Para equilibrar la mente racional y emocional y, por tanto, dejar aflorar la mente sabia se trabajan tres habilidades «qué»: observar, describir y participar y tres habilidades «cómo»: no emitir juicios, unicidad mental y hacer lo que funciona.

\section{B. Habilidades de efectividad interpersonal}

Los sujetos con personalidad límite suelen experimentar una disregulación interpersonal; sus relaciones son caóticas, inestables, problemáticas, y les causan sufrimiento. Además, tienen serias dificultades para tolerar el abandono y controlar sus respuestas al mismo, que muchas veces suponen formas de agresión o autoagresión. En este modulo el objetivo que se plantea a los pacientes es "ser eficaz interpersonalmente». En este contexto, "ser eficaz» implica conseguir los objetivos en una relación, por ejemplo, obtener ayuda, y hacerlo de forma que no dañemos la relación con la otra persona ni pongamos en juego nuestro respeto personal.

La primera parte del módulo trata de que los pacientes analicen las situaciones y determinen los objetivos a lograr en las mismas. La segunda parte analiza los factores que contribuyen a la efectividad interpersonal y aquellos que interfieren en la misma. La tercera parte está dedicada a analizar aspectos que hay que considerar antes de pedir algo, expresar una opinión o decir que no. En la última parte se tratan las habilidades específicas, por ejemplo, mantener la posición mediante el disco rayado, utilizar un enfoque cortés y sosegado o no amenazar. Estas habilidades se trabajan idealmente por medio de role playing de situaciones hipotéticas o situaciones reales planteadas por los pacientes que puedan resultar problemáticas. Se proporciona además, una lista de ejemplos para practicar las habilidades de eficacia interpersonal en la vida cotidiana.

\section{Habilidades de regulación de las emociones}

Desde la perspectiva de la TDC, la disregulación emocional es un aspecto nuclear del trastorno límite de la personalidad, y la aplicación de técnicas que permitan un mejor manejo de las emociones es uno de los aspectos centrales de la psicoterapia.

Es común que el paciente con un trastorno de personalidad límite se sienta abrumado por una serie de emociones intensas, variables y desagradables; estas personas experimentan con frecuencia ira, frustración, tristeza y ansiedad y encuentran muy pocos recursos personales para manejar esos estados emocionales, que les resultan intolerables. Algunas conductas disfuncionales características del TLP, por ejemplo, los gestos autolíticos o el consumo de sustancias, son intentos de soluciones conductuales a esas emociones intolerables.

En este módulo, resulta especialmente importante trabajar de una forma validante. Los sujetos con TLP muchas veces se exigen no sentir, o se sienten profundamente inadecuados por sentir lo que están sintiendo. De hecho, gran parte del sufrimiento que estas personas experimentan tiene su origen en las respuestas secundarias (por ejemplo, vergüenza o ansiedad) ante emociones primarias. El paciente debe aprender a observar su respuesta emocional de una forma consciente y no sentenciosa. El terapeuta anima a los pacientes toleren sus emociones pero que sean capaces de cambiar la respuesta emocional y conductual que dan a ellas, que en muchos casos es dañina y causa más sufrimiento.

En este módulo, por tanto, se aprende a identificar y nombrar emociones y a reconocer la función que éstas cumplen en el entorno. Se adoptan, además, técnicas destinadas a reducir la vulnerabilidad asociada a un estilo de vida poco saludable respecto al sueño o la alimentación. Como forma de intervenir en la respuesta emocional, se ensayan técnicas para aumentar la conciencia y frecuencia de las emociones positivas, por ejemplo por medio de actividades agradables, y para reducir el impacto de las emociones negativas, por ejemplo, cam- 
biando la respuesta que se da a ellas mediante la acción opuesta a la emoción.

\section{Habilidades de tolerancia al malestar}

Las habilidades de tolerancia al malestar, como las habilidades básicas de conciencia, están basadas en el mindfulness y las filosofías zen y budistas relacionadas con la aceptación. Lo importante, en este caso, no es evitar el dolor o el sufrimiento, sino aprender a manejarlo con habilidad. Así, desde esta perspectiva, el dolor es parte de la vida y su aceptación es parte de cualquier proceso de cambio.

Las estrategias propuestas en este módulo se dividen en dos bloques: habilidades de supervivencia a las crisis y habilidades de aceptación de la realidad.

Las habilidades de supervivencia a las crisis van dirigidas a aprender a tolerar el dolor de una situación dada sin recurrir a conductas que mantengan o aumenten en sufrimiento. Los pacientes con trastorno de personalidad límite suelen responder a las situaciones dolorosas por medio de una respuesta impulsiva, por ejemplo, una autolesión. Estas respuestas suponen un alivio a corto plazo, pero empeoran el problema a largo plazo. Las técnicas que se enseñan en esta parte se dirigen a aprender a dar una nueva respuesta a las crisis: distraerse, proporcionarse estímulos positivos, mejorar el momento por medio de la imaginación y la relajación y pensar en los pros y los contras de conducta impulsiva.

Las habilidades de aceptación de la realidad tienen que ver con aceptar, de una forma no enjuiciadora, a uno mismo y a la situación actual tal como se presenta en el momento. Son técnicas para aprender a percibir y experimentarse a uno mismo y a su ambiente sin intentar cambiar o modificar nada. Estas técnicas incluyen la aceptación radical, dirigir la mente hacia la aceptación y cultivar la buena disposición. Se trabajan, con este propósito, ejercicios de conciencia de uno mismo y del entorno, de media sonrisa y de autobservación.

\section{Psicoterapia individual}

La psicoterapia individual es la piedra angular del tratamiento, que articula el resto de los componentes y sin la cual es imposible el avance. Antes del comienzo del tratamiento, y en una fase previa, el terapeuta ha de conseguir que el paciente esté motivado para iniciar la terapia y establecer un vínculo terapéutico. La psicoterapia individual dialéctico conductual se apoya en gran medida en la relación: el paciente debe concebir al terapeuta como alguien con el que se puede contar, y el terapeuta debe esforzarse por no repetir el ambiente patológico invalidante que el paciente ha experimentado a lo largo de su vida. Se ha de lograr un equilibrio (dialéctica) entre la aceptación de las emociones y las conductas y el esfuerzo por lograr su cambio.

Los pacientes límite tienen un gran número de conductas disfuncionales, muchas veces peligrosas que, además, suponen un obstáculo importante cuando se manifiestan en la terapia. En la TDC, por tanto, los objetivos principales se encaminan a mantener al paciente a salvo y asegurar su implicación en el tratamiento. Estos objetivos son, por orden de importancia (Linehan 1993a):

1. Reducir las conductas que atentan contra la vida del paciente: intentos de suicidio, pensamientos suicidas y autoagresiones.

2. Reducir conductas que interfieren gravemente en el tratamiento: no acudir a terapia, llegar tarde, acudir intoxicado, alejarse de las personas que proporcionan ayuda, no realizar las tareas terapéuticas o recurrir a la hospitalización como única forma de manejar una crisis.

3. Reducir conductas que interfieren de forma seria en la calidad de vida: uso de sustancias, trastornos de alimentación, absentismo laboral o escolar, descuido de enfermedades, no utilización de la red social de apoyo.

4. Incrementar las estrategias de afrontamiento destinadas a la regulación emo- 
cional: puesta en marcha de habilidades de conciencia, de relación interpersonal, de manejo emocional y de tolerancia al malestar.

Para determinar el foco de una terapia individual, se comienza evaluando la presencia o ausencia de estas conductas «objetivo» en la semana previa, y analizando los estímulos internos o externos que preceden y siguen a esas conductas objetivo. Descubrir las variables asociadas con esta conducta disfuncional determina a su vez qué conductas adaptativas alternativas deben ser integradas en el repertorio del paciente. Una vez se obtiene el repertorio de estas conductas, se comienza trabajando con aquéllas que resultan un problema tanto dentro de la sesión como fuera, como una forma de tratar los problemas en el presente. Así, el terapeuta dialéctico conductual puede analizar las reacciones emocionales exageradas del paciente basándose en las que éste exhibe en consulta.

Además de los expuestos como objetivos principales, existen otros patrones de conducta que pueden interferir gravemente con el progreso de la terapia. Estos patrones, considerados objetivos secundarios, se tratan muchas veces de forma paralela a los primarios, ya que están asociados a ellos. Por ejemplo, puede pueden hacerse intervenciones destinadas a que el paciente tolere el malestar intenso y extremo que precede a un intento de suicidio. Dos de los objetivos secundarios más frecuentes:

1. La vulnerabilidad emocional: hace referencia a la excesiva respuesta y reactividad hacia los estímulos del entorno. Los pacientes límite responden al menor contratiempo con un dolor insoportable, seguido de una pérdida de control conductual, por lo que intentan evitar a toda costa emociones desagradables, respondiendo ante las señales de malestar con retirada, huida o agresividad. Conductas como ataques al terapeuta, que interfieren en la terapia, están mantenidas por este patrón de evitación de las emociones negativas. En este caso, por ejemplo, el paciente puede probar a poner en marcha algunas de las habilidades de tolerancia al malestar.

2. La auto-invalidación: sería, según Linehan, la adopción o asunción como propias de las características de un entorno invalidante. Esto daría lugar a tres patrones principales de respuesta: a) la inhibición de experiencias y expresiones emocionales (ej. silencio y expresión seria como respuesta a la rabia) b) la percepción negativa de uno mismo (insultos, odio por sí mismo, falta de respeto) y c) exageración de la facilidad de resolución de problemas actuales (por ejemplo, pensar que se necesita simplemente fuerza de voluntad para no volver a auto mutilarse: "lo hago porque soy débil, no tengo carácter para evitarlo...») En la TDC el terapeuta tratará de ayudar a los pacientes a entender que su respuesta tiene sentido, ya que se trata de un intento desesperado por enfrentarse a las intensas emociones experimentadas. Los terapeutas comunican esta validación por medio de la escucha, el reflejo y aceptando el núcleo de verdad de las experiencias emocionales del paciente.

Alcanzar estos objetivos lleva, al menos, un año de tratamiento intensivo. En estadios posteriores los objetivos irán encaminados a conseguir una experiencia emocional completa, construir una vida propia y resolver los problemas familiares, laborales y sociales que se presenten y, finalmente, con lograr el crecimiento espiritual, superando el sentimiento de vacío (Gempeler, 2008).

\section{Consulta telefónica y reuniones de supervisión:}

Aunque lo central en la TDC es la terapia individual y el entrenamiento en habilidades, el tratamiento se completa con la consulta telefónica y las reuniones de supervisión entre los distintos terapeutas que llevan un caso.

La consulta telefónica tiene como objetivo atender las situaciones de crisis. En dicha consulta, el terapeuta tratará de promover el uso de 
técnicas específicas que puedan ser aplicadas para la situación dada; de esta forma, aumenta la generalización de las habilidades aprendidas a los ámbitos cotidianos. Cuando existan crisis en la relación terapéutica, la consulta telefónica puede usarse, además, para reparar esta relación sin necesidad de esperar a la próxima consulta. Recordemos que el paciente con trastorno de personalidad límite tiende a abandonar los tratamientos (y las relaciones) de una forma prematura, perpetuando la soledad y el sentimiento de rechazo e humillación que experimentan con el otro. Dado que la relación terapéutica muchas veces es la más importante de las relaciones, su permanencia es de suma importancia, y la llamada telefónica puede ser, en ese sentido, una estrategia crucial.

Las reuniones de supervisión son la manera que tiene la TDC de cuidar al terapeuta o terapeutas encargados de un caso. Además de la coordinación y el establecimiento de objetivos y estrategias comunes, estas reuniones tienen la función de servir de apoyo y ayudar a los profesionales implicados.

\section{Estilo terapéutico en la TDC}

Todas los enfoques terapéuticos llevan asociado un estilo particular: la neutralidad en las terapias dinámicas, la empatía en las terapias humanistas o el empirismo colaborador en las terapias cognitivo-conductuales. La TDC, a pesar de ser un tratamiento derivado de terapias cognitivo conductuales, tiene características propias en cuanto a cómo el terapeuta es dentro de la relación. Estas son algunas de los aspectos diferenciadores. (Linehan, 1993a, b).

1. La dialéctica: el terapeuta TDC constantemente busca el equilibrio entre tensiones, la síntesis de los opuestos (por ejemplo: aceptación vs. cambio, énfasis en los déficit vs énfasis en las fortalezas). Se busca, precisamente, que el paciente abandone el pensamiento dicotómico, aceptando que la realidad compleja y con múltiples contradicciones, y que el cambio es el estado natural. Para transmitir esta visión el terapeuta puede utilizar intervenciones paradójicas como la «técnica de abogado del diablo», en la que defiende el síntoma o conducta que realmente pretende cambiar. También se usan, con este propósito, metáforas, parábolas, mitos, cuentos y lemas. Uno de los más comunes, «aprender a hacer limonada cuando la vida te da limones», refleja precisamente este equilibrio entre aceptar la vida tal como se nos presenta y hacer un esfuerzo por cambiar las cosas.

2. La validación: es uno de los pilares fundamentales del tratamiento. El terapeuta se esforzará en transmitir a los pacientes que sus respuestas tienen sentido en su contexto. La validación se transmite mostrando un interés genuino por el paciente y manifestando interés y reflexión sobre lo que cuenta. Buscar las causas y los aspectos positivos de las acciones también son estrategias terapéuticas que pueden utilizarse para la validación. Por último, el terapeuta debe considerar al paciente como alguien similar a sí mismo, merecedor de respeto y con capacidad de cambio, y debe estar dispuesto a dar ánimo asumiendo esta perspectiva.

3. El énfasis en la solución de problemas: como complemento de la validación y aceptación, el terapeuta considerara todos los síntomas, incluso aquellos graves como las agresiones y los intentos de suicidio, como un problema a resolver. En este sentido, utilizará el análisis conductual y el análisis de soluciones como herramientas para buscar formas alternativas, y saludables, de resolver problemas. Las técnicas cognitivo conductuales clásicas, por ejemplo el uso de contingencias, pueden ayudar también a este propósito.

4. La comunicación empática e irreverente: la TDC combina una actitud realista, irreverente y paradójica frente a las conductas disfuncionales, por ejemplo las parasuicidas, con una notable calidez, flexibilidad, atención cercana y autorrevelación estratégica por parte del terapeuta. Una vez más, el terapeuta mantiene el equilibrio entre dos estilos terapéuticos aparentemente opuestos. 


\section{Evidencia científica}

La terapia dialéctico conductual fue la primera terapia específica para el TLP sobre la que se publicaron estudios de eficacia (Linehan, Armstrong, Suarez, Allmon y Heard, 1991). Se mostró que la TDC, en comparación con el tratamiento habitual (farmacoterapia, counselling y atención en urgencias), reducía de forma efectiva las conductas autolesivas, las hospitalizaciones y las visitas al servicio de urgencias y aumentaba la adherencia al tratamiento. Los logros continuaban un año después de la finalización del tratamiento. (Linehan, Heard y Armstrong, 1993). En este estudio, sin embargo, no hubo diferencias con el tratamiento habitual en la reducción del afecto negativo o las ideas de desesperanza. Estos datos se confirmaron en tres estudios posteriores, que tuvieron, no obstante, mayores tasas de abandono que el trabajo original (Bohus et al., 2004; Koons et al., 2001; Verheul et al., 2003).

Los resultados de eficacia han sido replicados recientemente por la autora (Linehan et al., 2006) en un diseño de seguimiento de dos años, donde muestra que la TDC es más eficaz que una terapia no conductual aplicada por psicoterapeutas expertos para reducir los intentos de suicidio, las automutilaciones, las visitas a urgencias y el número de hospitalizaciones de estos pacientes. Recientemente, algunos estudios muestran que versiones abreviadas de la TDC pueden resultar efectivas en la reducción de síntomas (Pasieczny y Coonor 2011; Stanley, Brodsky, Nelson y Dulit, 2007). A pesar de los resultados prometedores, aún son necesarios estudios de seguimiento a largo plazo, que comparen la TDC con otras terapias estructuradas y específicas para el TLP (Kliem, Kroguer y Kosfelder, 2010; Paris, 2010).

\section{Eficacia de la TDC en otras poblaciones}

Aunque empezó como un tratamiento específicamente diseñado para pacientes suicidas, la TDC se está aplicando en la actualidad a un amplio rango de patologías con resultados preliminares, pero prometedores (Robins y Chapman, 2004). Dado que la TDC se dirige específicamente a la disregulación emocional y sus consecuencias conductuales, fundamentalmente de tipo impulsivo o autodestructivo, no es extraño que pueda adaptarse para patologías que presenten, en alguna medida, este tipo de síntomas. En adolescentes, los datos experimentales muestran que, con ciertas adaptaciones, la TDC puede ser un tratamiento eficaz para reducir la ideación suicida, el tiempo de hospitalización y otros síntomas de TLP (Backer, Miller y van den Bosch, 2009). El tratamiento se ha adaptado también, con éxito, a adolescentes con trastorno bipolar (Goldstein, Axelson, Birmaher y Brent, 2007).

En los trastornos de la conducta alimentaria, las estrategias de la TDC pueden ayudar a reducir la frecuencia y gravedad de las purgas y atracones (Safer, Telch y Agras, 2001; Telch, Agras y Linehan, 2001). En la actualidad, se están desarrollando tratamientos dialéctico conductuales adaptados específicamente para la bulimia nerviosa cuyos resultados preliminares son alentadores (Hill, Craighead y Safer, 2011).

Una de las comorbilidades más frecuentes del trastorno límite de personalidad es el abuso de sustancias. La terapia dialéctico conductual ha sido adaptada por la autora y su grupo para facilitar la abstinencia y reducir la cantidad y gravedad de las recaídas, y los ensayos clínicos realizados parecen indicar que la TDC es un tratamiento útil también en estos casos (Dimeff y Linehan, 2008; Linehan et al., 1999; Linehan et al., 2002)

La TDC también se ha aplicado con éxito a ancianos deprimidos que se mostraron refractarios al tratamiento farmacológico. En estos pacientes, la psicoterapia grupal más el apoyo telefónico logró reducir significativamente los síntomas depresivos. (Lynch et al., 2003). Este hallazgo ha sido replicado recientemente con ancianos deprimidos con TLP comórbido (Lynch et al., 2007).

Por último, estudios recientes sugieren que el entrenamiento en habilidades de la TDC podría ayudar a reducir los síntomas de déficit de atención con hiperactividad en sujetos adultos (Hirvikoski et al., 2011). 


\section{Referencias}

Backer, H. S., Miller, A. L. y van den Bosch, L. M. C. (2009). [Dialectical behaviour therapy for adolescents; a literature review]. Tijdschrift Voor Psychiatrie, 51(1), 31-41.

Bohus, M., Haaf, B., Simms, T., Limberger, M. F., Schmahl, C., Unckel, C. y Linehan, M. M. (2004). Effectiveness of inpatient dialectical behavioral therapy for borderline personality disorder: a controlled trial. Behaviour research and therapy, 42(5), 487-499.

Crowell, S. E., Beauchaine, T. P. y Linehan, M. M. (2009). A biosocial developmental model of borderline personality: Elaborating and extending Linehan's theory. Psychological Bulletin, 135(3), 495-510. doi:10.1037/a0015616

Chapman, A. L. (2006). Dialectical behavior therapy: current indications and unique elements. Psychiatry (Edgmont (Pa.: Township)), 3(9), 62-68.

Dimeff, L. A., y Linehan, M. M. (2008). Dialectical behavior therapy for substance abusers. Addiction science y clinical practice, 4(2), 39-47.

Gempeler, J. (2008). Terapia conductual dialéctica. [Dialectical Behavioral Therapy]. Revista Colombiana de Psiquiatría, 1, 136-148.

Goldstein, T. R., Axelson, D. A., Birmaher, B., y Brent, D. A. (2007). Dialectical behavior therapy for adolescents with bipolar disorder: a 1-year open trial. Journal of the American Academy of Child and Adolescent Psychiatry, 46(7), 820-830.

Hayes, S. C., Villatte, M., Levin, M. y Hildebrandt, M. (2011). Open, aware, and active: contextual approaches as an emerging trend in the behavioral and cognitive therapies. Annual Review of Clinical Psychology, 7, 141-168.

Hill, D. M., Craighead, L. W. y Safer, D. L. (2011). Appetite-focused dialectical behavior therapy for the treatment of binge eating with purging: a preliminary trial. The International Journal of Eating Disorders, 44(3), 249-261.

Hirvikoski, T., Waaler, E., Alfredsson, J., Pihlgren, C., Holmström, A., Johnson, A.,... y Nordström, A.-L. (2011). Reduced ADHD symptoms in adults with ADHD after structured skills training group: results from a randomized controlled trial. $B e-$ haviour Research and Therapy, 49(3), 175-185.
Kliem, S., Kröger, C. y Kosfelder, J. (2010). Dialectical behavior therapy for borderline personality disorder: a meta-analysis using mixed-effects modeling. Journal of consulting and clinical psychology, 78(6), 936-951.

Koons, C. R., Robins, C. J., Lindsey Tweed, J., Lynch, T. R., Gonzalez, A. M., Morse, J. Q.,... y Bastian, L. A. (2001). Efficacy of dialectical behavior therapy in women veterans with borderline personality disorder. Behavior Therapy, 32(2), 371-390.

Linehan, M. M. (1993a). Cognitive-Behavioral Treatment of Borderline Personality Disorder (1. ${ }^{\mathrm{a}}$ Ed.). New York: The Guilford Press.

Linehan, M. M. (1993b). Skills Training Manual for Treating Borderline Personality Disorder (1. ${ }^{\mathrm{a}} \mathrm{Ed}$.). New York. The Guilford Press.

Linehan, M. M, Armstrong, H. E., Suarez, A., Allmon, D. y Heard, H. L. (1991). Cognitive-behavioral treatment of chronically parasuicidal borderline patients. Archives of General Psychiatry, 48(12), 1060-1064.

Linehan, M. M, Heard, H. L. y Armstrong, H. E. (1993). Naturalistic follow-up of a behavioral treatment for chronically parasuicidal borderline patients. Archives of General Psychiatry, 50(12), 971-974.

Linehan, M. M., Schmidt, H., 3rd, Dimeff, L. A., Craft, J. C., Kanter, J. y Comtois, K. A. (1999). Dialectical behavior therapy for patients with borderline personality disorder and drug-dependence. The American Journal on Addictions / American Academy of Psychiatrists in Alcoholism and Addictions, 8(4), 279-292.

Linehan, M. M, Dimeff, L. A., Reynolds, S. K., Comtois, K. A., Welch, S. S., Heagerty, P. y Kivlahan, D. R. (2002). Dialectical behavior therapy versus comprehensive validation therapy plus 12-step for the treatment of opioid dependent women meeting criteria for borderline personality disorder. Drug and Alcohol Dependence, 67(1), 13-26.

Linehan, M. M., Comtois, K. A., Murray, A. M., Brown, M. Z., Gallop, R. J., Heard, H. L.,... y Lindenboim, M. (2006). Two-year randomized controlled trial and follow-up of dialectical behavior therapy vs therapy by experts for suicidal behaviors and borderline personality disorder. Archives of General Psychiatry, 63(7), 757-766. 
Lynch, T. R., Morse, J. Q., Mendelson, T., y Robins, C. J. (2003). Dialectical behavior therapy for depressed older adults: a randomized pilot study. The American Journal of Geriatric Psychiatry: Official Journal of the American Association for Geriatric Psychiatry, 11(1), 33-45.

Lynch, T. R., Chapman, A. L., Rosenthal, M. Z., Kuo, J. R., y Linehan, M. M. (2006). Mechanisms of change in dialectical behavior therapy: theoretical and empirical observations. Journal of Clinical Psychology, 62(4), 459-480.

Lynch, T. R., Cheavens, J. S., Cukrowicz, K. C., Thorp, S. R., Bronner, L. y Beyer, J. (2007). Treatment of older adults with co-morbid personality disorder and depression: a dialectical behavior therapy approach. International Journal of Geriatric Psychiatry, 22(2), 131-143.

Paris, J. (2010). Effectiveness of different psychotherapy approaches in the treatment of borderline personality disorder. Current Psychiatry Reports, 12(1), 56-60.

Pasieczny, N., y Connor, J. (2011). The effectiveness of dialectical behaviour therapy in routine public mental health settings: an Australian controlled trial. Behaviour Research and Therapy, 49(1), 4-10.
Robins, C. J. y Chapman, A. L. (2004). Dialectical behavior therapy: current status, recent developments, and future directions. Journal of Personality Disorders, 18(1), 73-89.

Safer, D. L., Telch, C. F. y Agras, W. S. (2001). Dialectical behavior therapy for bulimia nervosa. The American Journal of Psychiatry, 158(4), 632-634.

Schnyder, U. (2009). Future perspectives in psychotherapy. European Archives of Psychiatry and Clinical Neuroscience, 259 Suppl 2, S123-128.

Stanley, B., Brodsky, B., Nelson, J. D. y Dulit, R. (2007). Brief Dialectical Behavior Therapy (DBTB) for Suicidal Behavior and Non-Suicidal Self Injury. Archives of Suicide Research, 11(4), 337-341.

Telch, C. F., Agras, W. S. y Linehan, M. M. (2001). Dialectical behavior therapy for binge eating disorder. Journal of Consulting and Clinical Psychology, 69(6), 1061-1065.

Verheul, R., Van Den Bosch, L. M. C., Koeter, M. W. J., De Ridder, M. A. J., Stijnen, T. y Van Den Brink, W. (2003). Dialectical behaviour therapy for women with borderline personality disorder: 12-month, randomised clinical trial in The Netherlands. The British journal of psychiatry: the journal of mental science, 182, 135-140. 
